\title{
REVIEW ARTICLE \\ Structural and functional properties of SARS-CoV-2 spike protein: potential antivirus drug development for COVID-19
}

Yuan Huang ${ }^{1}$, Chan Yang ${ }^{1}$, Xin-feng $\mathrm{Xu}^{1}$, Wei $\mathrm{Xu}^{1}$ and Shu-wen Liu ${ }^{1,2}$

Coronavirus disease 2019 is a newly emerging infectious disease currently spreading across the world. It is caused by a novel coronavirus, severe acute respiratory syndrome coronavirus 2 (SARS-CoV-2). The spike (S) protein of SARS-CoV-2, which plays a key role in the receptor recognition and cell membrane fusion process, is composed of two subunits, S1 and S2. The S1 subunit contains a receptor-binding domain that recognizes and binds to the host receptor angiotensin-converting enzyme 2, while the S2 subunit mediates viral cell membrane fusion by forming a six-helical bundle via the two-heptad repeat domain. In this review, we highlight recent research advance in the structure, function and development of antivirus drugs targeting the $S$ protein.

Keywords: SARS-CoV-2 virus; spike protein; antivirus drugs; antibodies; fusion inhibitors; host proteases inhibitors

Acta Pharmacologica Sinica (2020) 41:1141-1149; https://doi.org/10.1038/s41401-020-0485-4

\section{INTRODUCTION}

The epidemic of novel coronavirus disease 2019 (COVID-19) was caused by a new coronavirus occurred in December 2019, and now has spread worldwide and turned into a global pandemic [1]. The COVID-19 was quickly discovered to be caused by a coronavirus later named severe acute respiratory syndrome coronavirus 2 (SARS-CoV-2) [1], which belongs to the $\beta$ coronavirus family. It is the seventh known coronavirus to infect humans; four of these coronaviruses (229E, NL63, OC43, and HKU1) only cause slight symptoms of the common cold. Conversely, the other three, SARS-CoV, MERS-CoV, and SARSCoV-2, are able to cause severe symptoms and even death, with fatality rates of $10 \%, 37 \%$, and $5 \%$, respectively.

Although a large number of studies and clinical trials are being launched on COVID-19 around the world [2,3], no evidence from randomized clinical trials has shown that any potential therapy improves outcomes in patients [4]. As the epidemic spreads, it is critical to find a specific therapeutic for COVID-19, and vaccines targeting various SARS-CoV-2 proteins are under development.

SARS-CoV-2 is a single-stranded RNA-enveloped virus [5]. An RNA-based metagenomic next-generation sequencing approach has been applied to characterize its entire genome, which is $29,881 \mathrm{bp}$ in length (GenBank no. MN908947), encoding 9860 amino acids [6]. Gene fragments express structural and nonstructural proteins. The $\mathrm{S}, \mathrm{E}, \mathrm{M}$, and $\mathrm{N}$ genes encode structural proteins, whereas nonstructural proteins, such as 3-chymotrypsinlike protease, papain-like protease, and RNA-dependent RNA polymerase, are encoded by the ORF region [7].

A large number of glycosylated $S$ proteins cover the surface of SARS-CoV-2 and bind to the host cell receptor angiotensinconverting enzyme 2 (ACE2), mediating viral cell entry [8]. When the $S$ protein binds to the receptor, TM protease serine 2 (TMPRSS2), a type 2 TM serine protease located on the host cell membrane, promotes virus entry into the cell by activating the $S$ protein. Once the virus enters the cell, the viral RNA is released, polyproteins are translated from the RNA genome, and replication and transcription of the viral RNA genome occur via protein cleavage and assembly of the replicase-transcriptase complex. Viral RNA is replicated, and structural proteins are synthesized, assembled, and packaged in the host cell, after which viral particles are released (Fig. 1d) [9].

These proteins are critical to the viral life cycle and provide potential targets for drug therapies. For example, ACE2-based peptide, 3CLpro inhibitor (3CLpro-1), and a novel vinylsulfone protease inhibitor have been experimentally demonstrated to be effective against SARS-CoV-2 [10]. The SARS-CoV-2 S protein is highly conserved among all human coronaviruses (HCoVs) and is involved in receptor recognition, viral attachment, and entry into host cells. Due to its indispensable functions, it represents one of the most important targets for COVID-19 vaccine and therapeutic research. In this review, we summarize advances in research of the SARS-CoV-2 S protein and its therapeutic targeting.

\section{STRUCTURE OF THE S PROTEIN}

With a size of $180-200 \mathrm{kDa}$, the $S$ protein consists of an extracellular N-terminus, a transmembrane (TM) domain anchored in the viral membrane, and a short intracellular C-terminal segment [11]. $S$ normally exists in a metastable, prefusion conformation; once the virus interacts with the host cell, extensive structural rearrangement of the $S$ protein occurs, allowing the virus to fuse with the host cell membrane. The spikes are coated with polysaccharide molecules to camouflage them, evading surveillance of the host immune system during entry [12].

The total length of SARS-CoV-2 S is 1273 aa and consists of a signal peptide (amino acids 1-13) located at the N-terminus, the

\footnotetext{
${ }^{1}$ Guangdong Provincial Key Laboratory of New Drug Screening, School of Pharmaceutical Sciences, Southern Medical University, Guangzhou 510515, China and ${ }^{2}$ State Key Laboratory of Organ Failure Research, Southern Medical University, Guangzhou 510515, China

Correspondence: Wei Xu (xuwei3322@smu.edu.cn) or Shu-wen Liu (liusw@smu.edu.cn)
}

Received: 15 May 2020 Accepted: 15 July 2020

Published online: 3 August 2020 


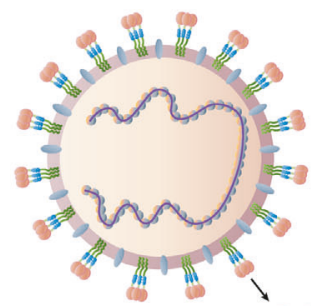

SARS-CoV-2

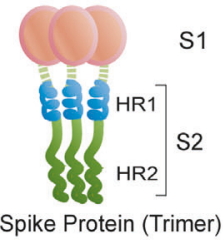

b
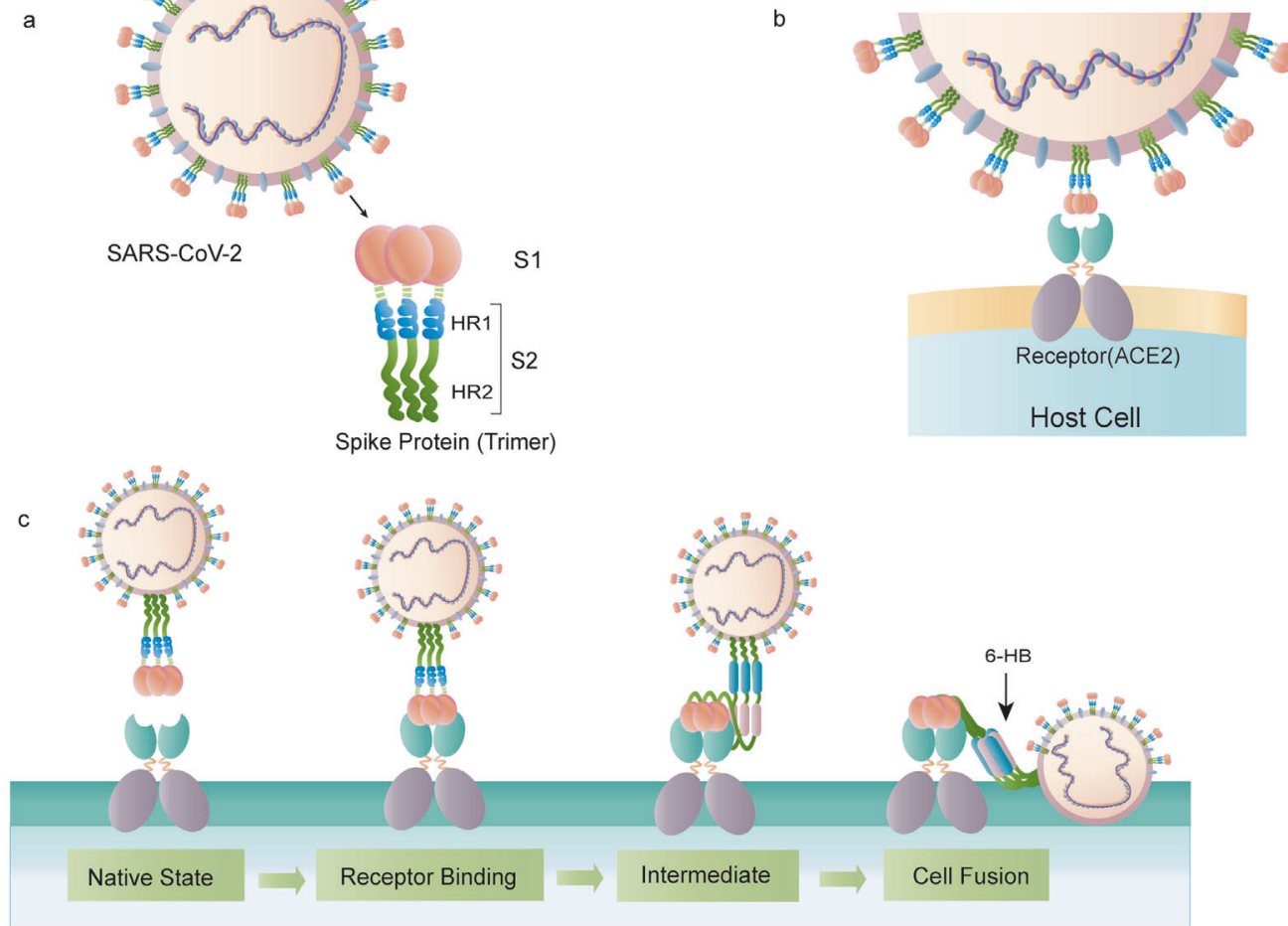

d

d
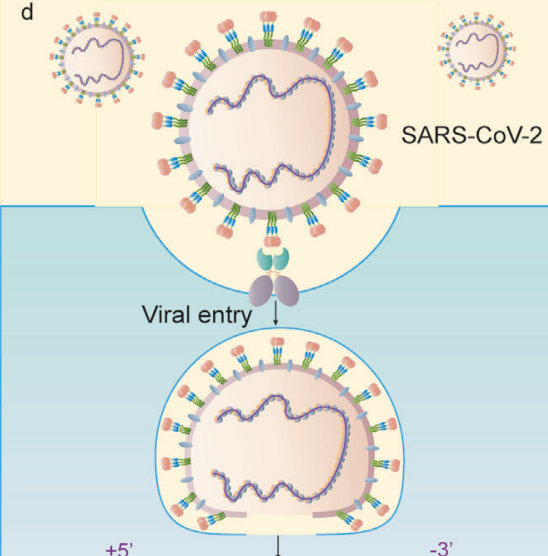

$5^{+2}$

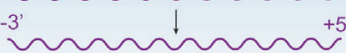

Replication and packaging
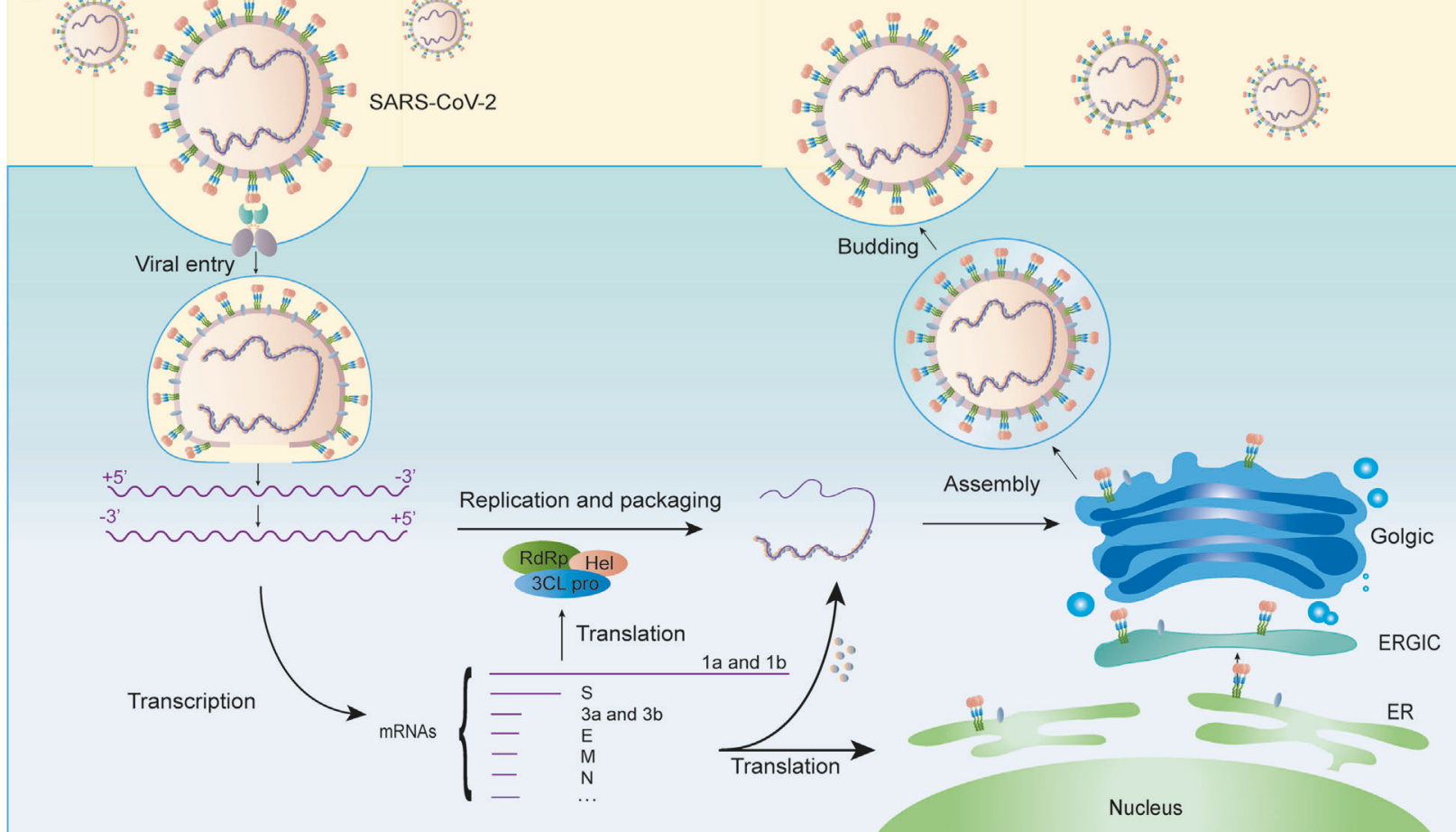

Fig. 1 Schematic of the SARS-CoV-2 $\mathbf{S}$ protein. a The schematic structure of the $\mathbf{S}$ protein. $\mathbf{b}$ The $\mathbf{S}$ protein binds to the receptor ACE2. $\mathbf{c}$ The binding and virus-cell fusion process mediated by the $S$ protein. $\mathbf{d}$ The life cycle of SARS-CoV-2 in host cells.

S1 subunit (14-685 residues), and the S2 subunit (686-1273 residues); the last two regions are responsible for receptor binding and membrane fusion, respectively. In the S1 subunit, there is an $\mathrm{N}$-terminal domain (14-305 residues) and a receptor-binding domain (RBD, 319-541 residues); the fusion peptide (FP) (788-806 residues), heptapeptide repeat sequence 1 (HR1) (912-984 residues), HR2 (1163-1213 residues), TM domain (1213-1237 residues), and cytoplasm domain (1237-1273 
a

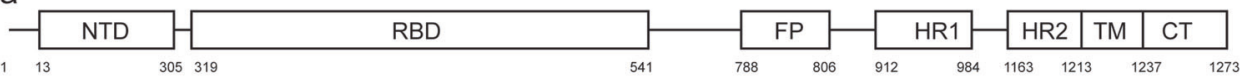

b

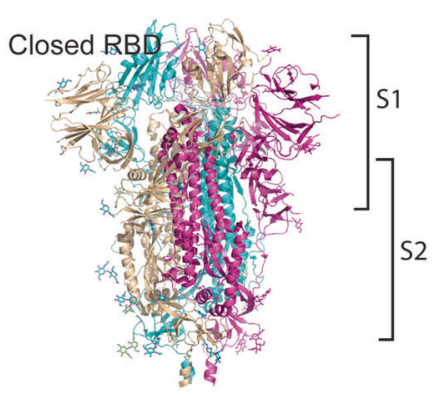

d

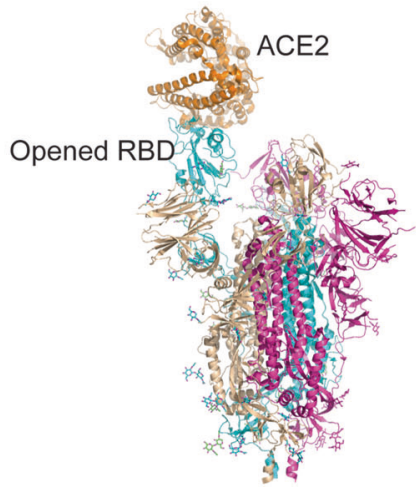

C

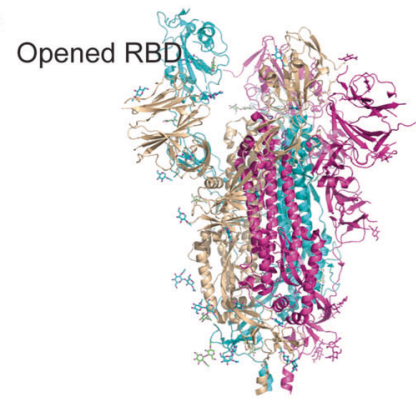

e

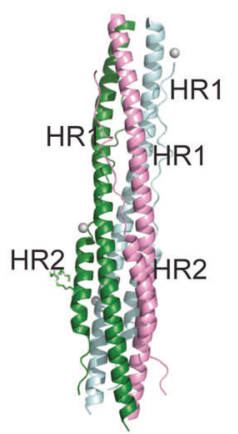

Fig. 2 Structure of the SARS-CoV-2 S protein. a Schematic representation of the SARS-CoV-2 spike. b-c The S protein RBD closed and opened status. d The S protein binds to ACE2 with opened RBD in the S1 subunit. e The six-helix structure formed by HR1 and HR2 of the S2 subunit.

residues) comprise the S2 subunit (Fig. 2a) [13]. S protein trimers visually form a characteristic bulbous, crown-like halo surrounding the viral particle (Fig. 1a). Based on the structure of coronavirus $S$ protein monomers, the S1 and S2 subunits form the bulbous head and stalk region [14]. The structure of the SARS-CoV-2 trimeric $S$ protein has been determined by cryo-electron microscopy at the atomic level, revealing different conformations of the S RBD domain in opened and closed states and its corresponding functions (Fig. 2b, c) $[15,16]$.

In the native state, the CoV $\mathrm{S}$ protein exists as an inactive precursor. During viral infection, target cell proteases activate the S protein by cleaving it into S1 and S2 subunits [17], which is necessary for activating the membrane fusion domain after viral entry into target cells [18]. Similar to other coronaviruses, the S protein of SARS-CoV-2 is cleaved into S1 and S2 subunits by cellular proteases, and the serine protease TMPRSS2 is used as a protein primer. Although the cleavage site of SARS-CoV is known, that of SARS-CoV-2 S has not yet been reported $[18,19]$.

Structure of the S1 subunit

The binding of virus particles to cell receptors on the surface of the host cell is the initiation of virus infection; therefore, receptor recognition is an important determinant of viral entry and a drug design target.

RBD situated in the $\mathrm{S} 1$ subunit binds to the cell receptor ACE2 in the region of aminopeptidase N. The S1 region contains the NTD and CTD, and atomic details at the binding interface demonstrate key residue substitutions in SARS-CoV-2-CTD. In addition, the SARS-CoV-2 S CTD binding interface has more residues that directly interact with the receptor ACE2 than does SARS-RBD (21 versus 17), and a larger surface area is buried with SARS-CoV-2 S CTD in complex with ACE2 than with SARS S RBD. Mutations of key residues play an important role in enhancing the interaction with ACE2. F486 in SARS-CoV-2, instead of 1472 in SARS RBD, forms strong aromatic-aromatic interactions with ACE2 Y83, and E484 in SARS-CoV-2-CTD, instead of P470 in SARS RBD, forms ionic interactions with $\mathrm{K} 31$, which leads to higher affinity for receptor binding than RBD of SARS-CoV (Fig. 2d) [15, 16, 20, 21].

The RBD region is a critical target for neutralizing antibodies (nAbs), and SARS-CoV-2 and SARS-CoV RBD are $~ 73 \%-76 \%$ similar in sequence. Nine ACE2-contacting residues in CoV RBD are fully conserved, and four are partially conserved. Analysis of the RBM (receptor-binding motif, a portion of RBD making direct contacts with ACE2) of SARS-CoV and SARS-CoV-2 revealed that most residues essential for ACE2 binding in the SARS-CoV S protein are conserved in the SARS-CoV-2 S protein. However, some studies showed that murine monoclonal antibodies (mAbs) and polyclonal antibodies against SARS-RBD are unable to interact with the SARS-CoV-2 $S$ protein, revealing differences in antigenicity between SARS-CoV and SARS-CoV-2 [20]. Similarly, a SARS-CoV RBD-specific antibody failed to block infection mediated by the $S$ protein of SL-CoV-SHC014 [22], which suggests that the S1 RBD may not be an ideal drug target due to the highly mutable characteristic of broad-spectrum anti-CoV drugs.

Structure of the S2 subunit

The S2 subunit, composed successively of a FP, HR1, HR2, TM domain, and cytoplasmic domain fusion (CT), is responsible for viral fusion and entry.

FP is a short segment of 15-20 conserved amino acids of the viral family, composed mainly of hydrophobic residues, such as glycine $(\mathrm{G})$ or alanine $(\mathrm{A})$, which anchor to the target membrane when the $S$ protein adopts the prehairpin conformation. Previous research has shown that FP plays an essential role in mediating 
membrane fusion by disrupting and connecting lipid bilayers of the host cell membrane [23].

HR1 and HR2 are composed of a repetitive heptapeptide: HPPHCPC, where $\mathrm{H}$ is a hydrophobic or traditionally bulky residue, $P$ is a polar or hydrophilic residue, and $C$ is another charged residue [24]. HR1 and HR2 form the six-helical bundle (6-HB) (Fig. 2e), which is essential for the viral fusion and entry function of the S2 subunit [13]. HR1 is located at the C-terminus of a hydrophobic FP, and HR2 is located at the N-terminus of the TM domain [25]. The downstream TM domain anchors the S protein to the viral membrane, and the S2 subunit ends in a CT tail [14].

RBD binds to ACE2, and S2 changes conformation by inserting FP into the target cell membrane, exposing the prehairpin coiledcoil of the HR1 domain and triggering interaction between the $\mathrm{HR} 2$ domain and HR1 trimer to form 6-HB, thus bringing the viral envelope and cell membrane into proximity for viral fusion and entry [26]. HR1 forms a homotrimeric assembly in which three highly conserved hydrophobic grooves on the surface that bind to HR2 are exposed. The HR2 domain forms both a rigid helix and a flexible loop to interact with the HR1 domain. In the postfusion hairpin conformation of CoVs, there are many strong interactions between the HR1 and HR2 domains inside the helical region, which is designated the "fusion core region" (HR1core and HR2core regions, respectively).

Targeting the heptad repeat (HR) has attracted the greatest interest in therapeutic drug discovery. The $S$ protein is an important target protein for the development of specific drugs, while the S1 RBD domain is part of a highly mutable region and is not an ideal target site for broad-spectrum antiviral inhibitor development [27]. In contrast, the HR region of the S2 subunit plays an essential role in $\mathrm{HCoV}$ infections and is conserved among HCoVs, as is the mode of interaction between HR1 and HR2 [28]. A synthetic peptide derived from the stem region of the ZIKV envelope protein was demonstrated in 2017 to potently inhibit infection by ZIKV and other flaviviruses in vitro [29], implying antiviral efficiency of peptides derived from conserved regions of viral proteins. Peptides derived from the HR2 region of class I viral fusion proteins of enveloped viruses competitively bind to viral HR1 and effectively inhibit viral infection [22]. Therefore, HR1 is a promising target for the development of fusion inhibitors against SARS-CoV-2 infection.

\section{FUNCTIONS OF THE S PROTEIN}

The $S$ protein on the surface of the virus is a key factor involved in infection. It is a trimeric class I TM glycoprotein responsible for viral entry, and it is present in all kinds of HCoVs, as well as in other viruses such as HIV (HIV glycoprotein 160, Env), influenza virus (influenza hemagglutinin, $\mathrm{HA}$ ), paramyxovirus (paramyxovirus $\mathrm{F}$ ), and Ebola (Ebola virus glycoprotein) [30]. Similar to other coronaviruses, the $S$ protein of SARS-CoV-2 mediates receptor recognition, cell attachment, and fusion during viral infection $[16,20,21,31-33]$.

The trimer of the $S$ protein located on the surface of the viral envelope is the basic unit by which the $S$ protein binds to the receptor $[16,33]$. The $S 1$ domain contains the $\mathrm{RBD}$, which is mainly responsible for binding of the virus to the receptor, while the S2 domain mainly contains the HR domain, including HR1 and HR2, which is closely related to virus fusion [34].

\section{Receptor binding}

As mentioned above, the SARS-CoV-2 S protein binds to the host cell by recognizing the receptor ACE2 [33]. ACE2 is a homolog of $A C E$, which converts angiotensin I to angiotensin 1-9 [35]. ACE2 is distributed mainly in the lung, intestine, heart, and kidney, and alveolar epithelial type II cells are the major expressing cells [36]. ACE2 is also a known receptor for SARS-CoV. The S1 subunit of the SARS-CoV $S$ protein binds with ACE2 to promote the formation of endosomes, which triggers viral fusion activity under low $\mathrm{pH}$ (Fig. 1a, b) [37].

Interaction between the $S$ protein and ACE2 can be used to identify intermediate hosts of SARS-CoV-2, as ACE2 from different species, such as amphibians, birds, and mammals, has a conserved primary structure [38]. Luan et al. compared the binding affinities between ACE2 and SARS-CoV-2 S from mammals, birds, snakes, and turtles and found that the ACE2 of Bovidae and Cricetidae interacted well with SARS-CoV-2 S RBD but that ACE2 from snakes and turtles could not.

The $S$ protein binds to ACE2 through the RBD region of the S1 subunit, mediating viral attachment to host cells in the form of a trimer [15]. SARS-CoV-2 S binds to human ACE2 with a dissociation constant $\left(K_{\mathrm{D}}\right)$ of $14.7 \mathrm{nM}$, though that of SARS-CoV S is $325.8 \mathrm{nM}$ [15], indicating that SARS-CoV-2 S is more sensitive to ACE2 than is SARS-CoV S. Through the identification of SARS-CoV2 proteins, researchers found $\sim 24 \%$ difference in S between SARSCoV-2 and SARS-CoV, whereas that of RBD is 23\% [39].

Viral fusion

Viral fusion refers to fusion of the viral membrane and host cell membrane, resulting in the release of the viral genome into the host cell. Cleavage of the SARS-CoV-2 S1 and S2 subunits is the basis of fusion. The $S$ protein is cleaved into two parts, the S1 subunit and S2 subunit, by host proteases, and the subunits exist in a noncovalent form until viral fusion occurs [40]. Researchers have found that the specific furin cleavage site is located in the cleavage site of SARS-CoV-2 but not in other SARSlike CoVs $[41,42]$. Mutation of the cleavage site in SARS-CoV-2 or SARS-like CoVs has revealed that the $S$ protein of SARS-CoV-2 exists in an uncleaved state but that the others are mainly in a cleaved state. SARS-CoV-2 S has multiple furin cleavage sites, which increases the probability of being cleaved by furin-like proteases and thereby enhances its infectivity $[43,44]$. The furin-like cleavage domain is also present in highly pathogenic influenza virus and is related to its pathogenicity, as observed in the avian influenza outbreak in Hong Kong in 1997 [45, 46]. In addition, host cell proteases such as TMPRSS2 are essential for $\mathrm{S}$ protein priming, and they have been shown to be activated in the entry of SARS-CoV and influenza A virus [18, 47, 48]. Another host cell protease that has been proven to cleave viral $S$ protein is trypsin [49]. In summary, the S protein of SARS-CoV-2 is similar to that of SARS-CoV, and host cell proteases are essential for promoting $\mathrm{S}$ protein cleavage of both SARS-CoV-2 and SARS-CoV. The presence of a specific furin cleavage site on SARS-CoV-2 S might be one reason that SARS-CoV-2 is more contagious than SARS-CoV.

The formation of 6-HB is essential for viral fusion. The FP in the $\mathrm{N}$-terminus of SARS-CoV-2 and the two HR domains on S2 is essential for viral fusion [50]. After cleavage of the $S$ protein, the FP of SARS-CoV-2 is exposed and triggers viral fusion. Under the action of some special ligands, the fusion protein undergoes a conformational change and then inserts into the host cell membrane (Fig. 1c) [51]. For example, the ligand for influenza $A$ virus is $\mathrm{H}^{+}$, while the ligand for HIV is a coreceptor such as CCR5 or CXCR4 [14]. The distance between the viral membrane and host cell membrane is shortened, and the HR1 domain of the S protein is in close proximity to the host cell membrane, whereas the HR2 domain is closer to the viral membrane side. Then, HR2 folds back to HR1, the two HR domains form a six-helix structure in an antiparallel format of the fusion core, the viral membrane is pulled toward the host cell membrane and tightly binds to it, and the two membranes fuse [52].

\section{POTENTIAL DRUGS TARGETING THE S PROTEIN}

The fundamental role of the $S$ protein in viral infection indicates that it is a potential target for vaccine development, 


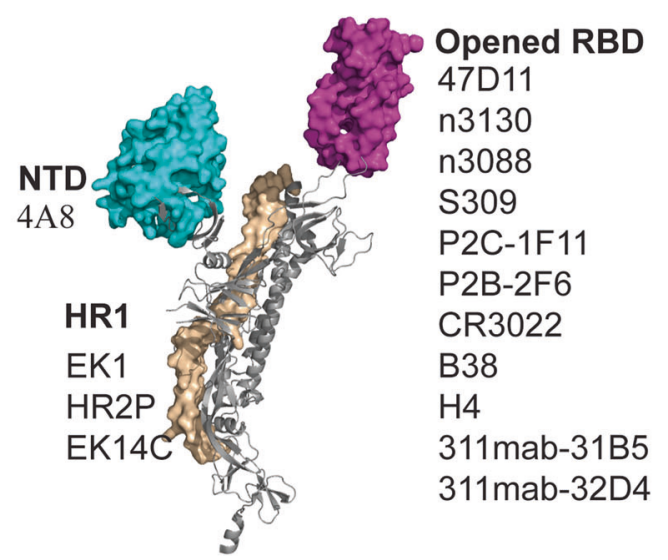

b

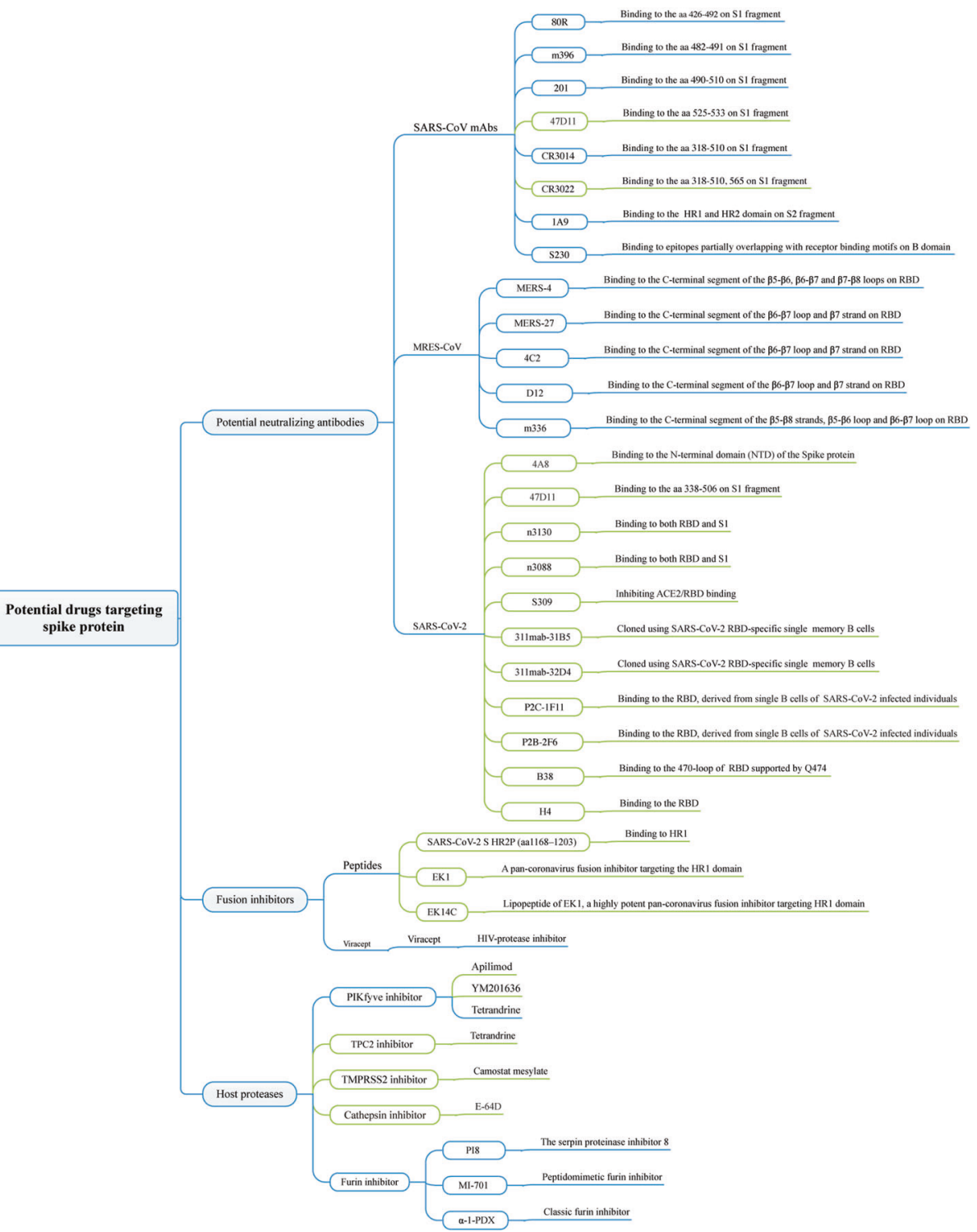

Fig. 3 Potential drugs targeting the SARS-CoV-2 S protein. a Potential mAbs targeting various epitopes of the S protein. $\mathbf{b}$ Summary of current SARS-CoV-2 inhibitors.

antibody-blocking therapy, and small molecule inhibitors. Considering the similarity with SARS-CoV and MERS-CoV, potential $\mathrm{nAbs}$ and inhibitors targeting SARS-CoV-2 $\mathrm{S}$ are summarized below (Fig. 3).
Antibodies based on the SARS-CoV-2 S protein

The $S$ protein is the main antigen component in all structural proteins of SARS-CoV-2. Unlike other functional proteins of SASCoV-2, it is responsible for inducing the host immune response, 
and nAbs targeting the $S$ protein can induce protective immunity against viral infection. Similar to SARS-CoV and MERS-CoV, research on nAbs of SARS-CoV-2 mainly includes mAbs, antigenbinding fragments, single-chain variable region fragments, and single-domain antibodies (Nbs), which target S1 RBD, S1-NTD, or S2 regions to prevent S2-mediated fusion [53, 54]. On the other hand, multiple SARS-CoV-2 vaccine types are under development, including RNA/DNA-based formulations, recombinant viral epitopes, adenovirus-based vectors, and purified inactivated virus [55].

The sequence and striking structural similarity between the SARS-CoV-2 and SARS-CoV $S$ proteins emphasize the close relationship between these two viruses, which provides the possibility to treat COVID-19 with antibodies targeting the SARSCoV S protein [56]. Compared with SARS-CoV-2 RBD, SARS-CoV-2 interacts with hACE2 via the C-terminal domain (SARS-CoV-2-CTD), showing higher affinity for receptor binding. RBD can induce highly potent $\mathrm{nAb}$ responses and has the potential to be developed as an effective and safe subunit vaccine against SARS-CoV-2. SARS-CoV S polyclonal antibodies obtained from immunized mice completely inhibited the invasion of SARS-CoV SMLV (murine leukemia virus), whereas the invasion rate of SARSCoV-2 S-MLV was reduced to $\sim 10 \%$ [20]. The polyclonal anti-SARS S1 antibody T62 inhibits the entry of SARS-CoV S but not that of SARS-CoV-2 S pseudovirus particles [49]. Consistently, recent studies have reported similar results, showing that three SARS RBD-directed mAbs, S230, m396, and 80R, were unable to bind to SARS-CoV-2 RBD [16, 20, 21].

On the other hand, several mAbs have shown promising results in neutralizing SARS-CoV-2. CR3022, a SARS-CoV-specific human $\mathrm{mAb}$, binds potently with SARS-CoV-2 $\left(K_{\mathrm{D}}\right.$ of $6.3 \mathrm{nM}$, measured by BLI in OctetRED96), suggesting that CR3022 has the potential to be developed as candidate therapeutic, alone or in combination with other nAbs, for the prevention and treatment of SARS-CoV-2 infection [57]. A mAb targeting S1 prepared from immunized transgenic mice expressing human Ig variable heavy and light chains has recently been shown to neutralize both SARS-CoV-2 and SARS-CoV infections via an unknown mechanism that is independent of the blockade of RBD-hACE2 interaction [58]. Recently, many human blocking mAbs (311mab-31B5, 311mab32D4, 47D11, n3130, n3088, S309, P2C-1F11, P2B-2F6, B38, H4) have been successfully cloned from single memory $B$ cells from recovered COVID-19 patients [58-63]. These mAbs specifically bind to SARS-CoV-2 S to effectively neutralize infection. In addition, sera from SARS patients during rehabilitation or animals specifically immunized with SARS-CoV S1 may cross-neutralize SARS-CoV-2 and reduce S protein-mediated SARS-CoV-2 entry (Fig. 3) [18].

Fusion inhibitors

The stability of the SARS-CoV-2 S protein is lower than that of SARS-CoV $S$ [42]. The mapping of multiple $S$ sequences of the subgenus Sarbecovirus underscores that the S2 fusion region is more conserved than the $\mathrm{S} 1$ subunit and that the $\mathrm{S} 1$ subunit is more exposed at the viral surface [16]. The SARS-CoV S2 subunit plays a key role in mediating virus-cell fusion and its integration into host cells, where HR1 and HR2 interact to form 6-HB, thus enabling the virus to bind to and fuse with the cell membrane [28].

Sequence alignment shows that SARS-CoV-2 HR2 has the same sequence as SARS-CoV HR2. Therefore, SARS-CoV-2 HR2P (1168-1203 residues) was designed to inhibit SARS-CoV-2 fusion and entry into a target cell. Surprisingly, HR2P showed inhibitory activity against SARS-CoV-2 S-mediated fusion and SARS-CoV-2 pseudovirus, with $\mathrm{IC}_{50}$ values of 0.18 and $0.98 \mu \mathrm{M}$, respectively [13]. Notably, EK1 is a pancoronavirus fusion inhibitor targeting the HR1 domain of HCoV S [22]. The X-ray crystal structure of the 6-HB core of the SARS-CoV-2 S2 subunit HR1 and HR2 domains has been solved, indicating that several mutant residues in the HR1 region may be related to enhanced interaction in the HR2 region [64]. Subsequently, EK1C4, a lipopeptide derived from EK1, was generated and verified to inhibit SARS-CoV-2 S-mediated cell-cell fusion. As expected, the entry of SARS-CoV-2 S pseudovirus was also inhibited by EK1C4, with an $\mathrm{IC}_{50}$ of $15.8 \mathrm{nM}$, 149-fold more potent than the original EK1 peptide. Another sequence-based lipopeptide fusion inhibitor, IPB02, potently inhibits SARS-CoV-2 S protein-mediated cell-cell fusion and pseudovirus infection [65].

In addition to peptide fusion inhibitors, nelfinavir mesylate (Viracept), a currently prescribed anti-HIV protease inhibitor, suppresses both SARS-CoV-2 S and SARS-CoV S-mediated cell-cell fusion. Viracept is the first reported small molecule fusion inhibitor in addition to peptide fusion inhibitors. Moreover, nelfinavir may inhibit the function of TMPRSS2 involved in activation of the $S$ protein [66]. This discovery makes possible clinical applications of anti-SARS-CoV-2 therapeutics, especially in the early stage of infection.

Protease inhibitors targeting SARS-CoV-2 S cleavage sites SARS-CoV-2 entry requires cleavage of the $S$ protein at the $S 1 / S 2$ and S2 sites. Proteolysis by TMPRSS2 and cathepsin B and L plays an important role in priming SARS-CoV-2 S for entry. Camostat mesilate is a potent serine protease inhibitor of TMPRSS2. Utilizing research on the SARS-CoV and SARS-CoV-2 cell entry mechanism, it has been demonstrated that SARS-CoV-2 cellular entry can be blocked by camostat mesilate $[18,67]$. There are currently five clinical trials registered to evaluate the efficacy of camostat mesilate (ClinicalTrials.gov Identifier: NCT04321096, NCT04353284, NCT04338906, NCT04355052, NCT04374019). In addition, cathepsins in lysosomes are crucial for SARS-CoV entry via endocytosis. $\mathrm{E}-64 \mathrm{~d}$, an inhibitor of cathepsin L, blocks infection with SARS-CoV and SARS-CoV-2 PsV [68-70]. Future trials with COVID-19 patients may help to confirm the efficacy of E-64d therapy.

Phosphatidylinositol 3-phosphate 5-kinase (PIKfyve) is the main enzyme synthesizing PI(3,5)P2 in early endosomes [71]. Apilimod, a potent inhibitor of PIKfyve35, can significantly reduce the entry of SARS-CoV S pseudovirus into 293/hACE2 cells via early endosomes in a dose-dependent manner [49]. Treating 293/ hACE2 cells with another PIKfyve inhibitor, YM201636 [72], also had a similar effect. Moreover, a major downstream effector of PI $(3,5) P 2$, two-pore channel subtype 2 (TPC2) [73], is important for SARS-CoV-2 entry, and tetrandrine (an inhibitor of TPC2) inhibits the activity of SARS-CoV-2 S pseudovirus.

Furin (proprotein convertase (PC) subtilisin kexin 3, PCSK3), as a member of the PC family, catalyzes the hydrolysis of peptide and protein substrates at paired basic residues [74]. Strikingly, SARSCoV-2 S harbors a furin cleavage site (682-685 residues) at the S1/ S2 boundary, which may increase the efficiency of SARS-CoV-2 transmission [75]. The furin-like cleavage site in the $S$ protein of SARS-CoV-2 may have implications for the viral life cycle and pathogenicity. Therefore, furin inhibitors can be used as a drug therapy for SARS-CoV-2 [41]. Patent literature since 1994 describes the use of furin or its inhibitors in the treatment of diseases, and some furin inhibitors that have been reported, including a-1-PDX (a1-antitrypsin Portland) [76], hexa-D-arginine(D6R) [77], serpin proteinase inhibitor 8 (PI8) [78], and a peptidomimetic furin inhibitor [79].

\section{FUTURE ASPECTS OF THE DEVELOPMENT OF ANTIVIRUS DRUGS TARGETING THE SARS-COV-2 S PROTEIN}

The SARS-CoV-2 $S$ protein binds to the host cell receptor and induces virus-cell membrane fusion, which plays a vital role in the process of virus invasion. Moreover, the high affinity between the $\mathrm{S}$ protein and ACE2 increases the infectivity of SARS-CoV-2. Mammals including pangolins, pets (dogs and cats), and members of Cricetidae may be important for determining key residues for association with S from SARS-CoV and SARS-CoV-2 [80]. Further 
understanding of the structure and function of SARS-CoV-2 S will allow for additional information regarding invasion and pathogenesis of the virus, which will support the discovery of antiviral therapeutics and precision vaccine design.

Structural information will also assist in evaluating mutations of the SARS-CoV-2 S protein and will help in determining whether these residues have surface exposure and map to known antibody epitopes of S proteins from other coronaviruses. In addition, structural knowledge ensures that the proteins produced by constructs are homogeneous and participate in the prefusion conformation, which should maintain the most neutralizationsensitive epitopes when used as a candidate vaccine or B-cell probe for isolating neutralizing human mAbs. Furthermore, atomic-level details will enable the design and screening of small molecules that inhibit fusion. Since SARS-CoV-2 and SARS-CoV RBD domains share $75 \%$ amino acid sequence identity, future work will be necessary to evaluate whether any of these Abs neutralize newly emerged coronavirus. Overall, interaction between the S protein of SARS-CoV-2 and ACE2 should be further studied to contribute elucidation of the mechanism of SARS-CoV-2 infection. Similarly, focusing on high expression of the $S$ protein or its receptor binding region is also of great significance for the development of vaccines.

The S2 subunit of SARS-CoV-2 shows $88 \%$ sequence homology with the SARS-CoV S2 domain and is structurally conserved. Therefore, the development of antibodies targeting this functional motif may cross-bind and neutralize these two viruses and related CoVs. Antiviral peptides prevent SARS-CoV-2 membrane fusion and can potentially be used for the prevention and treatment of infection. It is worth mentioning that EK1C4, which targets the highly conserved HR1 domain of the S2 subunit, is expected to have therapeutic potential against SARS-CoV-2. More importantly, EK1C4 can be used as a nasal drop, which increases its medicinal properties, it possesses a high genetic barrier to resistance, and does not easily induce drug-resistant mutations. On the other hand, peptide fusion inhibitors may not be widely used clinically and have low bioavailability. Therefore, the development of oral small molecule fusion inhibitors is a major direction.

In the course of virus epidemics, the ability to adapt to external pressure is an important factor affecting the spread of the virus. Regarding the envelope S protein, recombination or mutation in the gene of its RBD can occur to promote transmission between different hosts and lead to a higher fatality rate [81]. Mutation of the aspartate (D) at position 614 to glycine (G614) results in a more pathogenic strain of SARS-CoV-2 [82], which makes it more difficult to develop antibodies or vaccines that target nonconservative regions. To effectively prevent disease, combinations of different mAbs that identify different epitopes on the SARS-CoV-2 $S$ surface can be assessed to neutralize a wide range of isolates, including escape mutants [83].

Currently, no specific therapeutic or prophylactic has been used clinically to treat or prevent SARS-CoV-2 infection. Nonspecific antiviral drugs, such as IFN-a (recombinant human IFN-a1b, IFNa2a), remdesivir, chloroquine, favipiravir, and lopinavir-ritonavir (Aluvia), have been clinically used to treat COVID-19 in China [84]. Nevertheless, NIAID-VRC scientists are developing a candidate vaccine expressing SARS-CoV-2 $S$ protein in mRNA vaccine platform technology. Clinical trials of the vaccine are expected in the coming months. Continued strengthening of the monitoring of the SARS-CoV-2 $\mathrm{S}$ protein is of great significance for subsequent new drug development and protection against COVID-19.

\section{ACKNOWLEDGEMENTS}

This project was supported by grants from Guangzhou Science and Technology Program (\#201803040006 to WX), the Fund of Natural Science Foundation of
Guangdong Province (\#2018A030313056 to WX), and grants from Major Scientific and Technological Projects of Guangdong Province (\#2019B020202002 to SWL).

\section{ADDITIONAL INFORMATION}

Competing interests: The authors declare no competing interests.

\section{REFERENCES}

1. Zhu N, Zhang D, Wang W, Li X, Yang B, Song J, et al. A novel coronavirus from patients with pneumonia in China, 2019. N Engl J Med. 2020;382:727-33.

2. Wu C, Liu Y, Yang Y, Zhang P, Zhong W, Wang Y, et al. Analysis of therapeutic targets for SARS-CoV-2 and discovery of potential drugs by computational methods. Acta Pharm Sin B. 2020;10:766-88.

3. Liu C, Zhou Q, Li Y, Garner LV, Watkins SP, Carter $\sqcup$, et al. Research and development on therapeutic agents and vaccines for COVID-19 and related human coronavirus diseases. ACS Cent Sci. 2020;6:315-31.

4. Sanders JM, Monogue ML, Jodlowski TZ, Cutrell JB. Pharmacologic treatments for coronavirus disease 2019 (COVID-19): a review. JAMA. 2020;323:1824-36.

5. Lu R, Zhao X, Li J, Niu P, Yang B, Wu H, et al. Genomic characterisation and epidemiology of 2019 novel coronavirus: implications for virus origins and receptor binding. Lancet. 2020;395:565-74.

6. Chen L, Liu W, Zhang Q, Xu K, Ye G, Wu W, et al. RNA based mNGS approach identifies a novel human coronavirus from two individual pneumonia cases in 2019 Wuhan outbreak. Emerg Microbes Infect. 2020;9:313-9.

7. Chan JF, Kok KH, Zhu Z, Chu H, To KK, Yuan S, et al. Genomic characterization of the 2019 novel human-pathogenic coronavirus isolated from a patient with atypical pneumonia after visiting Wuhan. Emerg Microbes Infect. 2020;9:221-36

8. Letko M, Marzi A, Munster V. Functional assessment of cell entry and receptor usage for SARS-CoV-2 and other lineage B betacoronaviruses. Nat Microbiol. 2020;5:562-9.

9. Fehr AR, Perlman S. Coronaviruses: an overview of their replication and pathogenesis. Methods Mol Biol. 2015;1282:1-23.

10. Morse JS, Lalonde $T, X u S$, Liu WR. Learning from the past: possible urgent prevention and treatment options for severe acute respiratory infections caused by 2019-nCoV. Chembiochem. 2020;21:730-8.

11. Bosch BJ, van der Zee R, de Haan CA, Rottier PJ. The coronavirus spike protein is a class I virus fusion protein: structural and functional characterization of the fusion core complex. J Virol. 2003;77:8801-11.

12. Watanabe Y, Allen JD, Wrapp D, McLellan JS, Crispin M. Site-specific glycan analysis of the SARS-CoV-2 spike. Science. 2020;369:330-3.

13. Xia S, Zhu Y, Liu M, Lan Q, Xu W, Wu Y, et al. Fusion mechanism of 2019-nCoV and fusion inhibitors targeting HR1 domain in spike protein. Cell Mol Immunol. 2020;17:765-7.

14. Tang $T$, Bidon $M$, Jaimes JA, Whittaker GR, Daniel $S$. Coronavirus membrane fusion mechanism offers a potential target for antiviral development. Antivir Res. 2020;178:104792.

15. Wrapp D, Wang N, Corbett KS, Goldsmith JA, Hsieh CL, Abiona O, et al. Cryo-EM structure of the 2019-nCoV spike in the prefusion conformation. Science. 2020;367:1260-3.

16. Walls AC, Park YJ, Tortorici MA, Wall A, McGuire AT, Veesler D. Structure, function, and antigenicity of the SARS-CoV-2 spike glycoprotein. Cell. 2020;181:281-92 e286.

17. Bertram S, Dijkman R, Habjan M, Heurich A, Gierer S, Glowacka I, et al. TMPRSS2 activates the human coronavirus $229 \mathrm{E}$ for cathepsin-independent host cell entry and is expressed in viral target cells in the respiratory epithelium. J Virol. 2013;87:6150-60.

18. Hoffmann M, Kleine-Weber $\mathrm{H}$, Schroeder S, Kruger N, Herrler T, Erichsen $\mathrm{S}$, et al. SARS-CoV-2 cell entry depends on ACE2 and TMPRSS2 and is blocked by a clinically proven protease inhibitor. Cell. 2020;181:271-80.e8.

19. Du L, Kao RY, Zhou Y, He Y, Zhao G, Wong C, et al. Cleavage of spike protein of SARS coronavirus by protease factor $X a$ is associated with viral infectivity. Biochem Biophys Res Commun. 2007;359:174-9.

20. Wang $Q$, Zhang $Y$, Wu L, Niu S, Song C, Zhang Z, et al. Structural and functional basis of SARS-CoV-2 entry by using human ACE2. Cell. 2020;181:894-904.e9.

21. Lan J, Ge J, Yu J, Shan S, Zhou H, Fan S, et al. Structure of the SARS-CoV-2 spike receptor-binding domain bound to the ACE2 receptor. Nature. 2020;581:215-20.

22. Xia S, Yan L, Xu W, Agrawal AS, Algaissi A, Tseng CK, et al. A pan-coronavirus fusion inhibitor targeting the HR1 domain of human coronavirus spike. Sci Adv. 2019;5:eaav4580.

23. Millet JK, Whittaker GR. Physiological and molecular triggers for SARS-CoV membrane fusion and entry into host cells. Virology. 2018;517:3-8. 
24. Chambers $P$, Pringle $C R$, Easton AJ. Heptad repeat sequences are located adjacent to hydrophobic regions in several types of virus fusion glycoproteins. J Gen Virol. 1990;71:3075-80.

25. Robson B. Computers and viral diseases. Preliminary bioinformatics studies on the design of a synthetic vaccine and a preventative peptidomimetic antagonist against the SARS-CoV-2 (2019-nCoV, COVID-19) coronavirus. Comput Biol Med. 2020;119:103670.

26. Xia S, Xu W, Wang Q, Wang C, Hua C, Li W, et al. Peptide-based membrane fusion inhibitors targeting HCoV-229E spike protein HR1 and HR2 domains. Int J Mol Sci. 2018;19:487. https://doi.org/10.3390/ijms19020487.

27. Lu G, Wang Q, Gao GF. Bat-to-human: spike features determining 'host jump' of coronaviruses SARS-CoV, MERS-CoV, and beyond. Trends Microbiol. 2015;23:468-78.

28. Liu S, Xiao G, Chen Y, He Y, Niu J, Escalante CR, et al. Interaction between heptad repeat 1 and 2 regions in spike protein of SARS-associated coronavirus: implications for virus fusogenic mechanism and identification of fusion inhibitors. Lancet. 2004;363:938-47.

29. Yu Y, Deng YQ, Zou P, Wang Q, Dai Y, Yu F, et al. A peptide-based viral inactivator inhibits Zika virus infection in pregnant mice and fetuses. Nat Commun. 2017;8:15672.

30. Weissenhorn W, Dessen A, Calder LJ, Harrison SC, Skehel JJ, Wiley DC. Structural basis for membrane fusion by enveloped viruses. Mol Membr Biol. 1999;16:3-9

31. Gui M, Song W, Zhou H, Xu J, Chen S, Xiang Y, et al. Cryo-electron microscopy structures of the SARS-CoV spike glycoprotein reveal a prerequisite conformational state for receptor binding. Cell Res. 2017:27:119-29.

32. Hulswit RJ, de Haan CA, Bosch BJ. Coronavirus spike protein and tropism changes. Adv Virus Res. 2016;96:29-57.

33. Yan R, Zhang Y, Li Y, Xia L, Guo Y, Zhou Q. Structural basis for the recognition of SARS-CoV-2 by full-length human ACE2. Science. 2020;367:1444-8.

34. Cui J, Li F, Shi ZL. Origin and evolution of pathogenic coronaviruses. Nat Rev Microbiol. 2019;17:181-92.

35. Donoghue M, Hsieh F, Baronas E, Godbout K, Gosselin M, Stagliano N, et al. A novel angiotensin-converting enzyme-related carboxypeptidase (ACE2) converts angiotensin I to angiotensin 1-9. Circ Res. 2000;87:E1-9.

36. Zhang $\mathrm{H}$, Penninger JM, Li Y, Zhong N, Slutsky AS. Angiotensin-converting enzyme 2 (ACE2) as a SARS-CoV-2 receptor: molecular mechanisms and potential therapeutic target. Intensive Care Med. 2020;46:586-90.

37. Shang J, Wan Y, Luo C, Ye G, Geng Q, Auerbach A, et al. Cell entry mechanisms of SARS-CoV-2. Proc Natl Acad Sci USA. 2020;117:11727-34.

38. Chen Y, Guo Y, Pan Y, Zhao ZJ. Structure analysis of the receptor binding of 2019nCoV. Biochem Biophys Res Commun. 2020;525:135-40.

39. Wan Y, Shang J, Graham R, Baric RS, Li F. Receptor recognition by the novel coronavirus from Wuhan: an analysis based on decade-long structural studies of SARS coronavirus. J Virol. 2020;94:e00127-20.

40. Tortorici MA, Walls AC, Lang $Y$, Wang $C$, Li Z, Koerhuis D, et al. Structural basis for human coronavirus attachment to sialic acid receptors. Nat Struct Mol Biol. 2019;26:481-9.

41. Coutard B, Valle C, de Lamballerie X, Canard B, Seidah NG, Decroly E. The spike glycoprotein of the new coronavirus 2019-nCoV contains a furin-like cleavage site absent in CoV of the same clade. Antivir Res. 2020;176:104742.

42. Rabaan AA, Al-Ahmed SH, Haque S, Sah R, Tiwari R, Malik YS, et al. SARS-CoV-2, SARS-CoV, and MERS-COV: a comparative overview. Infez Med. 2020;28:174-84.

43. Hasan A, Paray BA, Hussain A, Qadir FA, Attar F, Aziz FM, et al. A review on the cleavage priming of the spike protein on coronavirus by angiotensin-converting enzyme-2 and furin. J Biomol Struct Dyn. 2020;22:1-9.

44. Millet JK, Whittaker GR. Host cell proteases: critical determinants of coronavirus tropism and pathogenesis. Virus Res. 2015;202:120-34.

45. Claas EC, Osterhaus AD, van Beek R, De Jong JC, Rimmelzwaan GF, Senne DA, et al. Human influenza $A$ H5N1 virus related to a highly pathogenic avian influenza virus. Lancet. 1998;351:472-7.

46. Kido H, Okumura Y, Takahashi E, Pan HY, Wang S, Yao D, et al. Role of host cellular proteases in the pathogenesis of influenza and influenza-induced multiple organ failure. Biochim Biophys Acta. 2012;1824:186-94.

47. Heurich A, Hofmann-Winkler H, Gierer S, Liepold T, Jahn O, Pohlmann S. TMPRSS2 and ADAM17 cleave ACE2 differentially and only proteolysis by TMPRSS2 augments entry driven by the severe acute respiratory syndrome coronavirus spike protein. J Virol. 2014;88:1293-307.

48. Limburg $\mathrm{H}$, Harbig A, Bestle D, Stein DA, Moulton HM, Jaeger J, et al. TMPRSS2 is the major activating protease of influenza $A$ virus in primary human airway cells and influenza B virus in human type II pneumocytes. J Virol. 2019;93: e00649-19.

49. Ou X, Liu Y, Lei $X$, Li P, Mi D, Ren L, et al. Characterization of spike glycoprotein of SARS-CoV-2 on virus entry and its immune cross-reactivity with SARS-CoV. Nat Commun. 2020;11:1620.
50. Kawase M, Kataoka M, Shirato K, Matsuyama S. Biochemical analysis of coronavirus spike glycoprotein conformational intermediates during membrane fusion. J Virol. 2019;93:e00785-19.

51. Harrison SC. Viral membrane fusion. Virology. 2015;479-480:498-507.

52. Eckert DM, Kim PS. Mechanisms of viral membrane fusion and its inhibition. Annu Rev Biochem. 2001;70:777-810.

53. Dhama K, Sharun K, Tiwari R, Dadar M, Malik YS, Singh KP, et al. COVID-19, an emerging coronavirus infection: advances and prospects in designing and developing vaccines, immunotherapeutics, and therapeutics. Hum Vaccin Immunother. 2020;16:1232-8

54. Zheng $\mathrm{M}$, Song $\mathrm{L}$. Novel antibody epitopes dominate the antigenicity of spike glycoprotein in SARS-CoV-2 compared to SARS-CoV. Cell Mol Immunol. 2020;17:536-8

55. Gao Q, Bao L, Mao H, Wang L, Xu K, Yang M, et al. Rapid development of an inactivated vaccine candidate for SARS-CoV-2. Science. 2020;369:77-81.

56. Coleman CM, Liu YV, Mu H, Taylor JK, Massare M, Flyer DC, et al. Purified coronavirus spike protein nanoparticles induce coronavirus neutralizing antibodies in mice. Vaccine. 2014;32:3169-74.

57. Tian X, Li C, Huang A, Xia S, Lu S, Shi Z, et al. Potent binding of 2019 novel coronavirus spike protein by a SARS coronavirus-specific human monoclonal antibody. Emerg Microbes Infect. 2020;9:382-5.

58. Wang C, Li W, Drabek D, Okba NMA, van Haperen R, Osterhaus A, et al. A human monoclonal antibody blocking SARS-CoV-2 infection. Nat Commun. 2020;11:2251.

59. Chen XY, Li R, Pan ZW, Qian CF, Yang Y, You RR, et al. Human monoclonal antibodies block the binding of SARS-CoV-2 spike protein to angiotensin converting enzyme 2 receptor. Cell Mol Immunol. 2020;17:647-9.

60. Wu Y, Li C, Xia S, Tian X, Wang Z, Kong Y, et al. Identification of fully human single-domain antibodies against SARS-CoV-2. Cell Host Microbe. 2020;27:891-8. e5. https://doi.org/10.1101/2020.03.30.015990.

61. Pinto D, Park Y-J, Beltramello M, Walls AC, Tortorici MA, Bianchi S, et al. Structural and functional analysis of a potent sarbecovirus neutralizing antibody. bioRxiv. 2020;2020.04.07.023903. https://doi.org/10.1101/2020.04.07.023903.

62. Ju B, Zhang Q, Ge X, Wang R, Yu J, Shan S, et al. Potent human neutralizing antibodies elicited by SARS-CoV-2 infection. bioRxiv. 2020; https://doi.org/ 10.1101/2020.03.21.990770.

63. Chi X, Yan R, Zhang J, Zhang G, Zhang Y, Hao $M$, et al. A potent neutralizing human antibody reveals the $\mathrm{N}$-terminal domain of the Spike protein of SARSCoV-2 as a site of vulnerability. bioRxiv. 2020. https://doi.org/10.1101/ 2020.05.08.083964.

64. Xia S, Liu MQ, Wang C, Xu W, Lan QS, Feng SL, et al. Inhibition of SARS-CoV-2 (previously 2019-nCoV) infection by a highly potent pan-coronavirus fusion inhibitor targeting its spike protein that harbors a high capacity to mediate membrane fusion. Cell Res. 2020;30:343-55.

65. Zhu Y, Yu D, Yan H, Chong H, He Y. Design of potent membrane fusion inhibitors against SARS-CoV-2, an emerging coronavirus with high fusogenic activity. J Virol. 2020;94:e00635-20.

66. Musarrat F, Chouljenko V, Dahal A, Nabi R, Chouljenko T, Jois SD, et al. The antiHIV drug Nelfinavir Mesylate (Viracept) is a potent inhibitor of cell fusion caused by the SARS-CoV-2 Spike (S) glycoprotein warranting further evaluation as an antiviral against COVID-19 infections. J Med Virol. 2020;10.1002/jmv.25985.

67. Uno Y. Camostat mesilate therapy for COVID-19. Intern Emerg Med. 2020;1-2. https://doi.org/10.1007/s11739-020-02345-9. [Epub ahead of print].

68. de Wilde $A H$, Falzarano D, Zevenhoven-Dobbe JC, Beugeling C, Fett C, Martellaro $C$, et al. Alisporivir inhibits MERS- and SARS-coronavirus replication in cell culture, but not SARS-coronavirus infection in a mouse model. Virus Res. 2017;228:7-13.

69. Huang IC, Bosch BJ, Li WH, Farzan M, Rottier PM. Choe H. SARS-CoV, but not HCoV-NL63, utilizes cathepsins to infect cells-viral entry. Nidoviruses: toward control of Sars and other Nidovirus. Diseases. 2006;581:335-8.

70. Zhou N, Pan T, Zhang JS, Li QW, Zhang X, Bai C, et al. Glycopeptide antibiotics potently inhibit Cathepsin $L$ in the late endosome/lysosome and block the entry of Ebola virus, Middle East respiratory syndrome coronavirus (MERS-CoV), and severe acute respiratory syndrome coronavirus (SARS-CoV). J Biol Chem. 2016;291:9218-32.

71. Nelson EA, Dyall J, Hoenen T, Barnes AB, Zhou H, Liang JY, et al. The phosphatidylinositol-3-phosphate 5-kinase inhibitor apilimod blocks filoviral entry and infection. PLoS Negl Trop Dis. 2017;11:e0005540.

72. Hou JZ, Xi ZQ, Niu J, Li W, Wang X, Liang $C$, et al. Inhibition of PIKfyve using YM201636 suppresses the growth of liver cancer via the induction of autophagy. Oncol Rep. 2019;41:1971-9.

73. Sakurai $Y$, Kolokoltsov AA, Chen CC, Tidwell MW, Bauta WE, Klugbauer N, et al. Two-pore channels control Ebola virus host cell entry and are drug targets for disease treatment. Science. 2015;347:995-8.

74. Artenstein AW, Opal SM. Proprotein convertases in health and disease. N Engl J Med. 2011;365:2507-18. 
75. Huang C, Wang Y, Li X, Ren L, Zhao J, Hu Y, et al. Clinical features of patients infected with 2019 novel coronavirus in Wuhan, China. Lancet. 2020;395:497-506.

76. Yakala GK, Cabrera-Fuentes HA, Crespo-Avilan GE, Rattanasopa C, Burlacu A, George $\mathrm{BL}$, et al. FURIN inhibition reduces vascular remodeling and atherosclerotic lesion progression in mice. Arterioscler Thromb Vasc Biol. 2019;39:387-401.

77. Zhou M, Zhang Y, Wei H, He J, Wang D, Chen B, et al. Furin inhibitor D6R suppresses epithelial-mesenchymal transition in SW1990 and PaTu8988 cells via the Hippo-YAP signaling pathway. Oncol Lett. 2018;15:3192-6.

78. Leblond J, Laprise MH, Gaudreau S, Grondin F, Kisiel W, Dubois CM. The serpin proteinase inhibitor 8: an endogenous furin inhibitor released from human platelets. Thromb Haemost. 2006;95:243-52.

79. Lu Y, Hardes K, Dahms SO, Bottcher-Friebertshauser E, Steinmetzer T, Than ME, et al. Peptidomimetic furin inhibitor MI-701 in combination with oseltamivir and ribavirin efficiently blocks propagation of highly pathogenic avian influenza viruses and delays high level oseltamivir resistance in MDCK cells. Antivir Res. 2015;120:89-100.

80. Abidin AZ, DSouza AM, Nagarajan MB, Wang L, Qiu X, Schifitto G, et al Alteration of brain network topology in HIV-associated neurocognitive disorder: a novel functional connectivity perspective. Neuroimage Clin. 2018;17:768-77.

81. Wang C, Liu Z, Chen Z, Huang X, Xu M, He T, et al. The establishment of reference sequence for SARS-CoV-2 and variation analysis. J Med Virol. 2020;92:667-74.

82. Becerra-Flores M, Cardozo T. SARS-CoV-2 viral spike G614 mutation exhibits higher case fatality rate. Int J Clin Pract. 2020;6:e13525.

83. Zhou GY, Zhao Q. Perspectives on therapeutic neutralizing antibodies against the novel coronavirus SARS-CoV-2. Int J Biol Sci. 2020;16:1718-23.

84. Wang ML, Cao RY, Zhang LK, Yang XL, Liu J, Xu MY, et al. Remdesivir and chloroquine effectively inhibit the recently emerged novel coronavirus (2019nCoV) in vitro. Cell Res. 2020;30:269-71. 